\title{
CAUSES FOR AND PREVENTION OF FAILED BACK SURGERY SYNDROME
}

\author{
Avramov T., Sv. Kalevski \\ Department of Neurosurgery "St. Anna" District hospital, Medical university \\ "Prof. d-r Paraskev Stojanov" Varna
}

Reviewed by: Assoc. Prof. St. Djankov, MD, PhD

\section{SUMMARY}

Persisting recurrent low back pain after surgical treatment of lumbar disc herniation (LDH) is also known in the literature as failed back surgery syndrome. The incidence rate reported is 8 to $5 \%$. FBSS is attributable to various causes, among them being residual disc fragments, central or lateral stenosis, facet-syndrome, postoperative adhesions, damage of the spinal nerve root, infections, psychological factors. Generally, it is assumed that a good prognosis after re-operation correlates with correct diagnosis and adequate application in practice of major principles of microneurosurgery, particularly emptying the intervertebral space. The latter is an adequate guarantee to avoid FBSS in the postoperative period. Of 341 patients, operated for LDH in our clinic, 45(13.2\%) were with unsatisfying postoperative results. The aim of the present study is: 1) to identify the causes of recurrent low back pain and compare clinical and operative findings with those obtained through CT, MRI and myelography; 2) to outline some guide-lines for adequate surgical treatment of LDH in an attempt to avoid the complications in the postoperative period

Keywords: low-back pain, lumber disk herniation, failed back surgery

\section{INTRODUCTION}

Despite the remarkable progress in spine surgery in treatment of lumbar disc herniation, a significant number of cases with unsatisfactory postoperative results are reported in the literature under the name of "failed back surgery syndrome" (FBSS). The latter is characterized as persisting chronic recurrent low-back pain after lumbar discectomy. Slipman et al. (2002) find this concept inaccurate, as it includes heterogenous factors identified as causing recurrent pain in 15 to $30 \%$ of patients undergoing surgery. According to Law, the frequency of this syndrome varies between 8 and $25 \%$. The most frequent causes for this syndrome are generally divided into two groups: non-surgical, including arachnoiditis, epineural fibrosis, battered nerve root, facet syndrome, discitis, psychological factors etc., and surgery-related, such as central or lateral stenosis, residual disc fragments (also known as pseudorecurrence), segmental instability, wrong level of operation, traumatic rupture of the dural sac or nerve root (1-7,8,11,13-14).

Detailed physical and neurological evaluation, combined with contemporary neuroimaging methods, helps to determine the cause of recurrent pain in most of the cases (95\%). In $5 \%$ of the patients, however, the causes remain unknown (7-9,12,15-17).

Address for correspondence:

T. Avramov, Dept. of Neurosurgery, Medical University, 55 Marin

Drinov St, BG-9002 Varna, BULGARIA
The aim of this study is to determine the causes of recurrent pain syndrome and neurological disorders in patients operated for lumbar disc herniation, and outline recommendations for prevention of FBSS.

\section{MATERIALS AND METHODS}

A retrospective study covered 396 patients operated for lumber disk herniation at the Clinic of Neurosurgery in Varna for the period 1997-2005. Unsatisfactory outcome from surgery was reported for 83 cases, of which 57 were re-operated at least once, and other 26 were given conservative treatment. The average age of patients was 46.6 years (range 16-69). In an attempt to elucidate the causes that account for FBSS, all patients were investigated neurologically and using neuroimaging methods (CT scanning, magnetic resonance imaging), and myelography. Of the patients with identified etiology of FBSS, 43 patients were re-operated once, 9 patients were re-operated twice, and 2 patients - three times. More than three re-operations were performed on 3 patients. Surgical procedures included 22 interlaminotomies and foraminotomies, accomplished in combination with medial facetectomy and osteophytectomy; 12 hemilaminectomies and 23 laminectomies were performed on patients with identified different kinds of stenosis, facet-syndrome, fibrosis, residual disc fragments, fistulas, infections etc. 


\section{RESULTS}

The period before re operation on the account of pain varied: it was up to 1 month - in 7 patients, up to 6 months - in 14 patients, up to 24 months - in 18 patients and more that 24 months -18 patients. Lateral stenosis was found in 12 patients $(21 \%)$, residual disc herniation - in 18 patients (31\%), and 13patients $(22.8 \%)$ had epidural fibrosis. In 4 patients $(7 \%)$ were found to have wound-related cerebrospinal fluid fistulas and pseudomeningocele. $\mathrm{Pa}-$ tients with wound infections were $5(8.8 \%)$, and 2 patients $(3.5 \%)$ had been operated at a wrong level. The cause of pain remained unidentified in 1 patient, 1 had a tumor (a meningeoma) at Th10, and one patient had epidural hematoma (Table 1.). thors report a considerable percentage of unsatisfactory postoperative results. Slipman has reported 250,000 laminectomies in patients with lumbar disc herniation performed in the US each year, of which 30 to 40 thousand do not lead to improvement after surgery and report chronic residual pain. Successful cases are reported to account for $49-90 \%$, and unsuccessful ones range from 10 to $50 \%$ of all cases (Burton, 1981; Kim - 1992). The most numerous series of FBSS were published by Burton - 850 cases, and Fritsch - 400 cases. Lateral stenosis as a leading cause for FBSS was pointed out in $58 \%$ of cases, and central stenosis - for $14 \%$. The percentage of total stenosis (foraminal, central and lateral) is $21.5 \%$ (Slipman et. al), and according Wagnespack et al. (2002) it is $29 \%$. Based on the clinical significance, other causes rank as follows: disk fragmenta-

Tabl. 1 .

\begin{tabular}{||c|c|c|c|c||}
\hline \multirow{2}{*}{ Causes of FBSS } & \multicolumn{4}{|c||}{ Authors } \\
\cline { 2 - 5 } & Burton et al. (1981) & Slipman et al. (2002) & Wagnespack et al.(2002) & Our results(2005) \\
\hline Lateral stenosis & 58 & 21,5 & 29 & 21 \\
\hline Central stenosis & $7-14$ & (total stenosis) & & 31 \\
\hline Fragmentation of the disc & & 21,5 & & \\
\hline Residual discal hernia & $12-16$ & 12,4 & 17 & 22,8 \\
\hline Arachnoiditis & $6-16$ & 0,5 & & \\
\hline Epidural fibrosis & $6-8$ & 14,5 & 14 & \\
\hline Spondylolisthesis & 1 & 1,6 & & 6,4 \\
\hline Facet syndrome & & 2,7 & & 8,8 \\
\hline Damaged nerve root & & 1,6 & & \\
\hline Discitis & & 0,5 & & \\
\hline Infections & & 5,6 & & \\
\hline Unknown & & & & \\
\hline \hline
\end{tabular}

Re-operation resulted in a significant regress of the pain syndrome in 17 patients, minimal residual lumber pain in 7 patients, and 5 patients reported significant pain. In 4 patients a minimal residual radicular syndrome was found, in other 4 - significant radicular pain. Minimal lumbar pain combined with radicular syndrome was found in 6 patients, while significant lumbar pain with radicular syndrome was found in 2 patients. Of the 57 patients, 31 were followed up for a period of 2 years after the last re-operation: 15 patients had no complaints, 10 recovered and reported with minimal complaints, and 6 patients experienced persisting neurological deficit and pain syndrome.

\section{DISCUSSION}

Although surgery for lumbar disc herniation is generally considered a feasible and effective intervention, many au- tion, residual disc herniation, spondylolisthesis, facet-syndrome, epidural fibrosis, damaged nerve root, arachnoiditis, infections and psychological factors. Table 1 shows most frequent causes of FBSS according to different authors:

Obviously, ignoring lateral stenosis as a surgery-related cause leads to persistent pain syndrome in a significant number of patients. According to our study, the syndrome was present in nearly one-third of the cases we analyzed. That is why we consider management of lateral stenosis as a mandatory objective of surgical intervention, together with lumbar disc herniation. Eliminating foraminal stenosis and facet-syndrome at a proper level through medial facetectomy and osteophytectomy seems to be the best way to prevent FBSS in the postoperative period.

Residual disc hernias are also among the most frequent causes of FBSS - they account for $31 \%$ of all cases we 
studied. Authors also report similar percentages for residual disc herniation. Microdiscectomy a modo Caspar\&Lowe, followed by inspection and removal of all fragments from the intervertebral space may guarantee prevention of postoperative pain syndrome and eventual re-operation. Epidural fibrosis is another major factor for FBSS. Burton et al. (1981) report 6-8 \% of cases with epidural fibrosis. Irrespective of our attempts to minimize bone and soft tissue resection, and restricted use of bipolar coagulation, combined with proper hemostasis and local application of fat graft, fibrosis occurred in $22.8 \%$ of our cases. Slipman et al (2002) divided causes of FBSS in two groups - surgical (stenosis, fragmentation of the disc, residual disc fragment, spondylolisthesis, spinal instability) and non-surgical (fibrosis, radiculopathy, facet-syndrome, damaged nerve root, discitis, arachnoiditis).Of the patients they present, 57\% suffered from spinal stenosis with persisting pain syndrome, $54 \%$ had ruptured herniated disc and $30 \%$ - residual disc fragment. Neurological disorders persisted even after re-operation. Causes cited for patients without improvement after the last operation include stenosis $(30 \%)$, residual disc fragment $(18 \%)$, disc fragmentation $(53 \%)$, and fibrotic changes $(56 \%)$. Etiology of pain was remained unidentified in $54.5 \%$ of the patients.

According to Long et. al. (1988), factors not related to surgery account for $83 \%$ of FBSS, and the ratio between surgical and non-surgical factors is $4.5: 1$. In contrast, Bernard et al. (1993) regard surgical factors as the most frequent causes, defining arachnoiditis and degenerative spondylosis as non-surgical etiologies.

Disputes in the literature on defining surgery and non-surgery related causes are attributable to the lack of objective criteria in defining non-surgical etiologies. Frymoeyr et al. (1991) divided surgical causes for FBSS into four categories according the period of their occurrence:

a) changes immediately after surgery - wrong diagnosis or technical mistakes;

b) early post-operative changes - infections, meningeal cysts;

c) intermediate post-operative changes - prolapse of residual disc fragment, damage of nerve root, arachnoiditis;

d) late post-operative changes - stenosis, instability.

In our study, we identified almost all causes for FBSS reported by authors, and the results obtained are similar to those reported by Wagnespack et al. (2002). The percentage of iatrogenic factors was also high - $33 \%$. We also found lumbar disc herniation on another level in 4 cases, infection of the wound - in 5 cases; rupture of the dural sac and nerve root damage - in 4 cases; wrong level of surgical intervention - in 1 case. Despite the perioperative application of antibiotics, postoperative infection seems to be an unavoidable problem, though the percentage is very low. Burton et al. (1988) recommend non-surgical methods for FBSS prevention, such as body weight reduction, remedial gymnastics, physiotherapy, tractions, extensions, transcutaneous neurostimulation, and pararadicular blockades. According to the majority of authors, the above mentioned surgical and non-surgical causes of FBSS can be controlled through adequate preoperative diagnoses, selection of patients and observing some principles of operative treatment such as wide enough operative incision, minimal tissue trauma, use of operative microscope and microneurosurgery techniques, dry operative field, and perioperative antibiotic prophylaxis. $(2-9 ; 12-17)$ For the treatment of lumbar disc herniation in particular, surgical techniques recommended include microsurgical discectomy, emptying the interveretebral space from disc fragments, intraforaminal radicular decompression through foraminotomy and medial facetectomy. Extradural fat graft placement helps to prevent severe fibrosis and soft-tissue adhesions.

\section{CONCLUSION}

The concept of FBSS has been used in the literature during the last 20 years in an attempt to group together direct and indirect factors causing persistent or residual pain syndrome in patients operated on for lumbar disc herniation. Despite the use of modern neuroimaging techniques for evaluation of lumbar disc herniation and application of sophisticated principles of microneurosurgery, postoperative results are still unsatisfactory. FBSS could be avoided through:

1. correct topic diagnosis;

2. localizing the level;

3. microdiscectomy and emptying the intervertebral space from fragments;

4. foraminotomy;

5. medial facetectomy;

6. extradural fat graft placement;

7. perioperative antibiotic prophylaxis.

Abiding to these principles during surgical treatment of lumbar disc hernia is a good guarantee to prevent postoperative complications.

\section{REFERENCES}

1. Albeck MJ,Kjaer L et al: MRI, CT and myelography in the diagnosis of recurrent lumbar disc herniation. Acta Neurochir (Wien)1990, 102:122-126

2. Bernard TN: Repeat lumbar spine surgery. Spine 1993, 18: 2196-2200

3. Bnef HV, Van d Reis W: Low back pain. Prim Care Clin North Amer 1996, 23: 345-364

4. Burton CV: Full thickness autogenous fat grafts in the prevention of epidural fibrosis. Neurosurgery, $1984,5: 1-5$

5. Burton CV: Avoiding "Failed Back Surgery Syndrome". In Lumbar Spine Surgery, Sec. Ed.,1988, Williams \& Wilkins, p.331-341.

6. Burton CV,Kirkaldy-Willis WH et al: Causes of failure of surgery on the lumbar spine. Clinical Orthopaedics, 1981, 157:191-199

7. Fritsch E,Heisel J: Ursachen fur Fehlschlage nach lumbalen Bandscheibenoperationen. Orthop Praxis 1992, 2:96-104 
8. Fritsch EW,Heisel J,Rupp S: The FBSS: reasons, intraoperative findings and long term results:a report of 182 operative treatments. Spine 1996, 21:626-633

9. Frymoyer JW, Matteri RE, HanleyEN et al: Failed lumbar disc surgery requiring second operation. A long-term follow-up study. Spine 1978, 3:7-11

10. Keane GP: FBSS. The low back pain handbook Philadelphia. Mosby;1997, 269-281

11. Kim SS, Michelsen CB: Revision surgery for failed back surgery syndrome. Spine 1992 17, 8:957-960

12. Law JD, Lehman RAW, Kirsch WM et al: Reoperation after lumbar intervertebral disc surgery. J Neurosurg 1978, 48:259-263

13. Long N,Filtzer DL et al: Clinical features of the failed back syndrome. J Neurosurg 1988, 69:61-71
14. North RB, Campbell JN, James CS et al: FBSS: 5-year follow-up in 102 patients undergo- ing repeated operations. Neurosurgery, 1991, 28, 5:685-691

15. Slipman CW, Shin CH, Rajeev KP et al: Etiologies of FBSS. Amer Acad Pain Med, 2002 3: 200-207

16. Striffeler H, Groger U, Reulen HJ: "Standard " microsurgical lumbar discectomy vs. "conservative" microsurgical discectomy. Acta Neurochir (Wien) 1991, 112(1-2):62-64

17. Wagnespack A, Schofferman J, Slosar P et al: Etiology of long- term failures of lumbar Spine Surgery. Pain Med 2002, 3, 1:18 - 22 